\title{
TELMA RECA EN LA GESTIÓN ESTATAL DE LA SANIDAD ARGENTINA (1930-1948)
}

\author{
Karina Inés Ramacciotti \\ Universidad de Buenos Aires- CONICET y Universidad de Quilmes \\ karinaramacciotti@gmail.com \\ ORCID iD: https://orcid.org/0000-0002-6724-3926
}

Recibido: 10 marzo 2016; Aprobado: 6 abril 2017.

Cómo citar este artículo/Citation: Ramacciotti, Karina Inés (2018), “Telma Reca en la gestión estatal de la sanidad argentina (19301948)", Asclepio, 70 (1): p211. https://doi.org/10.3989/asclepio.2018.04

RESUMEN: Este artículo aporta nuevos datos sobre la médica argentina Telma Reca (1904-1979), particularmente en relación con su inserción en ámbitos académicos y en la gestión estatal a partir de los años treinta del siglo XX. Nos focalizaremos en su trayectoria profesional desde que obtuvo su doctorado en la Universidad de Buenos Aires (1932) hasta que se retiró de la División de Maternidad e Infancia del Departamento Nacional de Higiene en 1948. Durante ese período aplicó en la gestión estatal sus investigaciones sobre el estudio de las condiciones sociales de la delincuencia juvenil y de la educación. Su perspectiva crítica, lejos de implementar medidas punitivas generadoras de una mayor exclusión social, buscó la integración de los menores en la comunidad por medio de políticas educativas y sanitarias.

PALABRAS CLAVE: Médicas argentinas; Políticas sanitarias; Políticas educativas; Integración social- organismos estatales.

\section{TELMA RECA, AN ARGENTINE PHYSICIAN IN THE STATE (1930-1948)}

ABSTRACT: This article will reconstruct the biography of the Argentine physician Telma Reca (1904-1979) who managed to get involved in academic fields and the state administration in the 30's. We will address ourselves to tracking her professional career from her PhD degree in Medicine (1932) until her withdrawal from the División de Maternidad e Infancia del Departamento Nacional de Higiene (Maternity and Childhood Division of the National Hygiene Department) in 1948. During this period, she focused her research on the study of the social conditions of juvenile delinquency on the public administration. With a critical eye, far from social exclusion and punitive measures, she managed to promote social integration through health and education policies.

KEY WORDS: Argentine Physician; Educational Policies; Public Health Policies; Social Integration- Government Agencies. 


\section{INTRODUCCIÓN}

Este artículo aporta nuevos datos sobre la médica argentina Telma Reca (1904-1979) quien logró una inserción en instancias académicas y en la gestión pública a partir de los años treinta del siglo pasado. Con ese objetivo se indaga en su recorrido académico y en su paso por organismos estatales desde que obtuvo su doctorado en la Universidad de Buenos Aires (UBA) (1932) hasta que se retiró de la División de Maternidad en Infancia del Departamento Nacional de Higiene (DNH) en 1948. Durante ese período volcó sus investigaciones sobre el estudio de las condiciones sociales de la delincuencia juvenil a la esfera estatal por medio de su rol como Jefa de las Divisiones de "Segunda Infancia y Niños Enfermos Anormales Necesitados" de la Dirección de Maternidad e Infancia del DNH. En esos años intentó convertir a la escuela pública en un centro de observación, investigación e individualización tanto de las conductas "antisociales" que pudieran conducir a la delincuencia como a la diferenciación de aquellos niños considerados anormales. Su mirada, lejos de buscar la exclusión social y la implementación de medidas punitivas, buscó integrarlos a la sociedad por medio de instituciones, que contaran con profesionales capacitados, que pudieran contemplar las diferencias y lograr su "adaptación". Desde el inicio de sus investigaciones se orientó hacia un enfoque social y, más particularmente, hacia el estudio que podía ejercer la educación y las intervenciones profesionales en la inclusión social.

Este trabajo pretende aportar al estudio de una veta, hasta el momento no explorada, del pensamiento Reca: como sus saberes profesionales, ligados a sus investigaciones sobre la criminalidad y la importancia de la escuela para detectar conductas "antisociales", se engarzaron con los saberes estatales, a partir de su gestión en la Dirección Maternidad e Infancia. Los trabajos publicados sobre Reca se han centrado en su descollante papel en la creación de la carrera de Psicología de la UBA en 1957, y en sus participaciones en relación con el psicoanálisis en la Argentina en los años sesenta (Plotkin, 2003). Otros estudios hicieron foco en sus conferencias sobre la educación sexual y sus actividades ligadas a la divulgación (Queirolo, 2013), y sobre sus trabajos sobre la historia de la criminología y la psicología (Cesano, 2015; Stagno, 2011; Fendrik, 2006; Dagfal, 2009; Talak, 2005). Este artículo propone una aproximación a las ideas y acciones de Reca en el ámbito de su accionar como una médica con un acendrado perfil social y que tuvo una activa participación en las agencias sanitarias del Estado. De esta manera, nos permite pensar que, a pesar de que las mujeres encontraban dificultades para insertarse en la educación superior y en los organismos estatales, algunas de ellas, como el caso de Reca, se esforzaron por demostrar y difundir sus ideas, siguiendo los lineamientos que exigía su campo profesional.

En función de este interés se analizarán algunos de sus múltiples registros -tesis doctoral, artículos científicos, libros, memorias estatales, aplicaciones a becas- y se intentará dar repuestas a las siguientes cuestiones: el papel otorgado a la escuela, el papel asignados a los docentes y a las visitadoras sociales; y las formas de intervención del Estado, por medio de sus técnicos, instituciones y leyes, para mejorar el sistema educativo "normal y especial".

\section{LOS AÑOS FORMATIVOS}

Nacida en 1904, Telma Reca obtuvo su título de doctor de la UBA en 1932 con una tesis titulada Delincuencia Juvenil en Estados Unidos y Argentina la cual recibió el premio "Eduardo Wilde". Su director fue el psiquiatra Nerio Rojas, profesor de Medicina Legal. El jurado estuvo compuesto por un catedrático del área de la Higiene Social Alberto Zwanck, Profesor Titular de Higiene y Medicina Social, y por los psiquiatras Osvaldo Loudet, Profesor de Clínica Psiquiátrica, y José Belbey, Profesor de Medicina Legal, todos de la misma casa de estudios. La tesis fue elaborada luego de una estancia de diez meses en 1930 en los Estados Unidos. Ese viaje fue medular en su experiencia de vida y en sus posteriores orientaciones; ella lo calificó, en su libro de corte autobiográfico De la vida norteamericana (1932), como "una sorpresa para mí. No fue el EE.UU. de los libros, del cine, de conversaciones con los viajeros entusiastas, con los conocidos norteamericanos. No fue el Estados Unidos loado por los amigos y escarnecido por los enemigos. Fue otro. Y otro tan diferente de mi panorama interior que hube de ir cambiando lentes espirituales, buscando foco mental, para verlo todo" (Reca, 1932 a, p.5).

El Vassar College de Nueva York le ofreció una beca, por intermedio del Instituto Cultural Argentino Norteamericano (ICANA), para estudiar en el John Hopkins Hospital aspectos ligados al bienestar y a la higiene infantil. Durante los diez meses que duró su estancia de investigación visitó cortes juveniles en Nueva York, Washington y Vassar, la Judge Baker Foundation y estuvo en contacto con Ann Platt, la Probation Officer, quien estaba a cargo de algunos menores. Esa experiencia le aportó riquísimos estímulos, que le permitieron recabar una voluminosa y 
actualizada información sobre la interpretación de la práctica y la doctrina norteamericana sobre el tema; observó las estrategias por medio de las cuales se resolvía la delincuencia e inclinó su profesión a la psicología, entendida como una disciplina "alejada de los tecnicismos del laboratorio y la clínica y más abocada al estudio de los resortes mentales de la conducta" (Reca, 1932 b, pp.86-87).

En su tesis doctoral destacó que la Corte Juvenil de Chicago fue la primera experiencia que intentó modificar el tratamiento judicial de los menores. Su creación se fundamentó en la oposición del confinamiento de niños y jóvenes en las cárceles junto a los adultos $y$, en particular, en una nueva sensibilidad hacia la infancia y la juventud que procuraba por parte de la justicia una actitud de tutela y protección (Stagno, 2011, p.350). En su tesis, Reca articuló las discusiones en boga ofrecidas por la medicina con las existentes, a la sazón, en materia jurídica. La perspectiva interdisciplinaria será un sesgo que acompañará a nuestra autora durante todo su derrotero profesional (vide infra). Según ella "necesitamos más centros donde psicólogos, pediatras, psiquiatras, educadores, trabajadores sociales, enfermeras de salud pública trabajen juntos aportando sus respectivas contribuciones" (Reca, 1937, p. 22).

A partir de esa experiencia, su formación académica estuvo muy ligada al medio norteamericano, pues realizó otros viajes de estudio a los EE.UU. financiados por la Fundación Rockefeller. Tuvo el respaldo de Bernardo Houssay, referente científico nacional dada su destacada trayectoria en el Instituto de Fisiología de Buenos Aires, y de Lewis Hackett, malariólogo y director de la oficina regional de la Fundación Rockefeller para el área del Río de la Plata y la región Andina entre 1941 a 1949. Entre 1942 a 1948 estuvo becada en tres oportunidades por dicha institución para estudiar el área de la psiquiatría infantil y la organización de las Child Guidance Clinics (Clínicas de conducta infantil) en diferentes ciudades de EE.UU. El estudio de esa experiencia y las referencias bibliográficas de ese origen fue una constante en su producción académica; también constituye un indicador de cómo, a partir de los años veinte del siglo XX, la medicina norteamericana fue cobrando un mayor protagonismo en Argentina. ${ }^{11}$

La entrada de las mujeres a la carrera de Ciencias Médicas no fue fácil; tuvieron que enfrentar dificultades tanto para su ingreso como para su permanencia. En el terreno profesional las mujeres se inclinaron, no siempre motivado por sus deseos personales, por las ramas de la medicina vinculadas a los cuidados de las mujeres y los niños (Lorenzo, 2016). Como se ha informado en trabajos anteriores (Ramacciotti y Valobra, 2011) las llamadas "primeras médicas" tuvieron un accionar ligado con el feminismo y el sufragismo; y mantuvieron una variedad de actividades políticas y sociales. En efecto, desde los años '30, conforme decreció la visibilidad de las militantes feministas entre las graduadas en medicina e incluso, en la militancia partidaria, muchas egresadas tuvieron un perfil más profesionalizado y buscaron una mayor inserción tanto en el ámbito universitario como en las agencias estatales. Estas mujeres lucharon por el reconocimiento de sus derechos en la jerarquía universitaria y administrativa del Estado. El caso de Telma Reca constituye un ejemplo, ya que no tuvo una militancia política explícita, pero sus inclinaciones estuvieron ligadas al campo de la izquierda. Algunos de sus escritos fueron publicados en revistas socialistas y comunistas tales como Vida Femenina o El Obrero Textil (Ramacciotti y Valobra, 2011; Guy, 2008; Norando, 2016) y algunos de sus proyectos, tal como la propuesta de ampliación edilicia del Centro de Psicología y Psiquiatría Infantil fue retomada por la bancada socialista en los años 40. Reca podría ser un ejemplo de las personas que el Partido Comunista denominó "compañeras de ruta" en los años '30; esto es integrantes de la sociabilidad intelectual antifascista y con simpatías hacia el comunismo (Pasolini, 2013 y Valobra, 2015).

Es interesante revisar las ideas presentadas por Reca en su tesis doctoral. La investigación tuvo como telón de fondo su preocupación por la delincuencia juvenil en Argentina y las consecuencias que traería para el futuro. Tal como sostiene Lucía Lionetti se buscaba la preservación del cuerpo social como un todo, más allá de la simple suma del bienestar de cada integrante individual. Las nociones de higiene y profilaxis se constituyeron en los pilares de esa intervención médica. La noción de higiene se asociaba al mejoramiento de las condiciones ambientales para evitar o minimizar la aparición de enfermedades o anomalías en la sociedad presente, y la de profilaxis, aludía a las intervenciones que buscaban desterrar en el presente los elementos perniciosos para la sociedad futura (Lionetti, 2011, p.34).

La tesis de Reca puede ser interpretada como una crítica a los efectos de la Ley del Patronato de Menores (1919), sancionada diez años antes. Dicho marco normativo reglamentó la pérdida o la suspensión de la patria potestad de los padres considerados incapaces de criar y educar a sus hijos menores de 18 años. En- 
tre las causas que podían motivar la pérdida de la capacidad jurídica y que ésta pasara al Estado nacional o provincial, se encontraban la incapacidad mental, la ebriedad consuetudinaria y las conductas "antisociales" de niños o jóvenes (Zapiola, 2010, p.119).

En su tesis Reca planteó que, si bien las conductas criminales podrían tener un sustrato biológico, tal cual lo planteaba la corriente lombrosiana; para ella el factor social, potenciado por los efectos de la urbanización y la industrialización, tenía un peso preponderante en las conductas delictivas de los menores; asociaba estas conductas particularmente a los sectores sociales pobres. Al respecto afirmaba: "debe repetirse, cuantas veces fuere necesario, que los menores delincuentes se reclutan entre los abandonados, en las clases sociales más pobres, en las viviendas hacinadas, en los medios donde la urbanización es más intensa" (Reca, 1932b, p. 17).

De esta manera, al poner el acento en las condiciones sociales, se apartaba de las argumentaciones que explicaban la criminalidad juvenil como un fenómeno individual o singular originado por factores biológicos, innatos e inmodificables y, lo analizaba como un fenómeno social pasible de ser modificado a partir de intervenciones públicas y privadas. Estas ideas se relacionaron con las de su colega Alicia Moreau de Justo quien, como lo demostró Adriana Valobra, tensionó con su tesis doctoral, el peso de lo biológico en el estudio de las conductas sociales (Valobra, 2012, p.139).

Reca no descartaba el peso de las causas biológicas, pero se concentraba en la posibilidad de modificar las cuestiones sociales para lograr la prevención de la delincuencia. El distanciamiento de su tesis respecto de las líneas hegemónicas de la ciencia ha sido documentado por Marcela Borinsky (2005, p.192). Efectivamente, Reca inició su tesis citando a Cesare Lombroso y su teoría de la degeneración para, rápidamente, distanciarse de esa postura y remarcar la necesidad de estudiar sus causas sociales.

Las medidas que proponía en "Delincuencia Juvenil en Estados Unidos y Argentina" se apartaron del criterio punitivo y se trasladaban al terreno de la reforma educacional y social apostando al papel del medio y a la modificación de las conductas por sobre cualquier herencia biológica (Talak, 2005, p. 592). A tono con el ideario higienista decimonónico y con los principios de la higiene mental, que habían llegado a la Argentina en la tercera década del siglo XX (Klappenbach, 1989; Talak, 2005), Reca planteaba que era necesario brindar protección prenatal a la madre embarazada y al niño tanto en la primera, como en la segunda infancia y en la edad escolar. Entre las acciones que sugería estaban la asistencia médica, la legislación social, la medicina preventiva y la educación pública. De esta batería de medidas, Reca se concentró en el rol que tendría la escuela en la "prevención de la delincuencia infantil y para contribuir a la pesquisa e identificación de esos sujetos no adaptables, y para discriminar y remediar, en lo posible, las causas de su inadaptación" (Reca, 1932b, p.19). Reca sostenía que "La escuela, como primera institución en que el niño sale del marco limitado de la familia y donde se le ofrecen numerosos estímulos nuevos, puede servir como precioso puesto de observación, piedra de toque, para descubrir aquellas anomalías y encauzarlas en un sentido provechoso" (Reca, 1932b, p. 19). La escuela era puesta como principal lugar de aprendizaje y, por lo tanto, abandonó la idea de que la educación se debería dar a través del contacto con los adultos. La escolarización, como ocurrió en otras latitudes, fue colocada como el lugar central de formación de los niños (Ariès, 1960).

Reca consideraba imprescindible que las escuelas contaran con un servicio médico escolar y con asistentes sociales. Como ha investigado Adrián Cammarota la salud escolar fue objeto de interés por los organismos sanitarios y educativos y, desde los años ' 20 , se promocionaron experiencias de inspección y relevamiento de datos en espacios escolares (Cammarota, 2016, pp. 36-38). Para Reca el servicio médico escolar debía cumplir la función de individualizar al niño abandonado, detectar las deficiencias en el estado físico, señalar deficiencias nutricionales, infecciones, y "taras hereditarias". Las asistentes sociales debían hacer cumplir las indicaciones médicas, intentar modificar el ambiente familiar en el sentido considerado conveniente, impulsar la difusión de los conocimientos necesarios para lograr la adquisición definitiva de normas y prácticas higiénicas, y realizar encuestas familiares y sociales (Reca, 1932b, p.233). Tal como lo indica Donzelot se fueron generando mecanismos de regulación y normalización de las familias. Las asistentes sociales, pedagogos y médicos infantiles fueron quienes se encargaron de clasificar a la infancia (Donzelot, 1998). Reca encasilló a la infancia utilizando las categorías de "normal o anormal". Los niños "normales" eran aquellos que estaban en la escuela, los "anormales" eran los pobres, desvalidos, peligrosos, o en peligro y quienes tenían muchas posibilidades de convertirse en delincuentes y de allí pasar a las instituciones estatales destinadas a los menores abandonados o delincuentes (Carli, 1992; Talak, 2005; Ramacciotti y Testa, 2014). 
Para Reca, la escuela debía contar con maestros dotados de conocimientos en psicología y pedagogía para atender las necesidades de "retardados, inestables y precoces". Si bien reconocía que para la primera categoría (que no definió a quienes incluía) existían niveles escolares especiales, la situación era diferente para las otras dos, para ella más preocupantes, ya que se caracterizaban por contar con alumnos "cuya vida posterior revela condiciones de inteligencia superiores al nivel medio donde actúan, que se singularizan durante la edad escolar por su conducta irregular y su desaplicación. Les faltaron estímulos eficaces para encadenar su atención y despertar su interés. Estos niños pueden incurrir en faltas, escapadas del colegio, vagancia, perturbación en clase o simplemente tienen un exceso de energía sin empleo" (Reca, 1932b, pp.21 y 22).

En síntesis, las escuelas, según Reca, eran un espacio central para detectar la delincuencia infantil. Allí se debía individualizar y brindar tratamiento al "individuo tarado"; descubrir "al predelincuente en el inadaptable"; asistir socialmente a la familia y detectar al niño en peligro moral para ejercer sobre él el patronato del Estado (Reca, 1932b, p.22). Los saberes de la psicología se entremezclaron con los educativos en una relación de complementación y de superposición a medida que se fueron conformando como campos profesionales en Argentina (Talak, 2005, p. 584).

Entonces, las problemáticas sociales se podrían revertir a partir del vínculo, en la escuela, de los abordajes profesionales y las responsabilidades estatales. En línea con lo que sostiene Donzelot (1998) la puesta bajo tutela de las familias pobres contó con la medicina y la psiquiatría como disciplinas hegemónicas que respaldaban esa maniobra. Luego, el desarrollo de las correspondientes carreras universitarias con el consiguiente surgimiento de profesionales específicos para la atención de niños, niñas y adolescentes, puso en manos de los trabajadores sociales y los pedagogos la intervención social sobre esta población que se apoyó en un saber proveniente de la piscología y de la pedagogía. De esta manera, Reca, a partir de su formación universitaria, su accionar en la gestión estatal y su intensa actividad vinculada a la divulgación de sus investigaciones, colaboró en la institucionalidad del saber clasificatorio sobre la infancia y en el impulso de espacio adecuados según sus capacidades. Reca no estuvo sola en esta empresa, su colega Carolina Tobar García estuvo en diferentes proyectos que impulsaron la nivelación, según capacidades, en la escuela pública. De hecho, Tobar García asumió, en 1942, como directora de la Escuela de Adaptación y allí dictó un curso destinado a los maestros comunes dispuestos a desempeñarse, a partir de 1948, en Escuelas Diferenciales de la Capital (Lardies González, 1974; Stagnaro, 2011; Sánchez, 2007; Ramacciotti y Testa, 2014).

\section{EJERCICIO PROFESIONAL}

\section{a. En el ámbito universitario}

En este apartado revisaremos las actividades de Reca en el ámbito universitario. Nos centraremos en aquellas experiencias que luego darán origen a las medidas que promovió durante su gestión en la administración estatal. Algo para destacar en Reca es que tanto en la universidad como en la gestión utilizó herramientas de las ciencias sociales. Esto es, relevamiento empírico a partir de encuestas sociales, análisis cualitativo, sistematización de la información, elaboración de informes y difusión de los resultados de sus investigaciones tanto en publicaciones científicas como en medios de divulgación popular.

En 1933, Reca siendo Jefe de Trabajos Prácticos en el Instituto de Higiene y Medicina Social de la Facultad de Ciencias Médicas de la UBA y desde su función de subdirectora del curso de Visitadoras Sociales, implementó una encuesta social en dos comedores escolares de la Capital Federal. El objetivo de la misma fue evaluar el estado nutricional y social de los niños para luego sugerir modificaciones. Como lo demuestra Lucía Aguilar las encuestas profesionales pueden ser interpretadas como parte del interés de la época por elaborar registros sociológicos que pudieran contribuir a la implementación de las políticas públicas (Aguilar, 2014, p. 231). Tanto la información presentada en los informes técnicos resultantes, como los diagnósticos y respuestas podrían orientar posibles cursos de acción de gobierno. En el caso aquí estudiado también nos muestra cómo estos relevamientos y los posteriores informes eran utilizados para abrir posibles instancias de inserción laboral dentro de las agencias del Estado a las mujeres y, más precisamente, a las visitadoras sociales. Como veremos más adelante, la implementación de encuestas sociales será una estrategia que Reca mantendrá durante su gestión en la administración estatal.

La encuesta de 1933, se vinculó con las reformas sociales que tendieron a impulsar cambios en las condiciones medioambientales de niños y adolescentes. Un año antes de esa investigación se había sancionado la Ley № 11.597 que estipuló la creación de los comedores escolares dependientes del Consejo 
Nacional de Educación. En estos espacios se brindaba un almuerzo completo con financiamiento estatal. Al año de la puesta en práctica de la ley, el diagnóstico al que arribó Reca fue desolador: faltaban lavatorios, el personal era exiguo, no existían médicos para realizar los exámenes clínicos periódicos ni los odontológicos. Asimismo, la ración diaria, según edad y peso, correspondía a un tercio de lo que se aconsejaba en la época, y las porciones eran poco variadas y equilibradas. El estado general de la alimentación era inferior al estándar ideal. Frente a este escenario sombrío, Reca y Zwanck, quien sumaba a sus antecedentes el de ser director del Instituto de Higiene y Medicina Social, miembro del cuerpo Médico Escolar y profesor de los cursos a las visitadoras de $\mathrm{Hi}$ giene, escribieron un artículo "Comedores escolares" publicado en la Semana Médica. Allí sostenían que sus funciones deberían ser ampliadas y reconocían su importancia como instrumento de política social, ya que constituían una herramienta más, dentro de un sistema de prestaciones amplias que aún debían completarse (Reca y Zwanck, 1934, p.12).

Sus propuestas para modificar el sistema fueron que los comedores escolares estuvieran vinculados a un organismo técnicamente capacitado y dotado para realizar el examen médico periódico. Se sugería que este organismo fuera la Clínica de Nutrición y Enseñanza de Hábitos de Salud organizada por la médica Perlina Winocur en 1928 con el fin de enseñar prácticas de higiene. Es decir, a la dependencia educativa sumaban una intervención médica activa. $A$ su vez planteaban que se debían impulsar campañas de divulgación dirigidas a los niños y también a las familias. Se señalaba que: "la ignorancia hace emplear el dinero de un presupuesto modesto, en forma que podría ser mejorada por un consejo". Estas actividades de mediación entre los consejos de la medicina, los comedores y las familias podrían ser realizadas a partir de la inclusión de visitadoras sociales dentro del equipo profesional de los comedores. De manera irónica sostenían que: "fondos había ya que existía una ley que los proveía pero que éstos no estaban dando un rendimiento efectivo" (Reca y Zwanck, 1934, p.12).

A partir de 1934, Reca trabajó en el Instituto de Pediatría del Hospital Nacional de Clínicas dependiente de la UBA. Allí creó el Consultorio de Higiene Mental que en los años '40 se transformó en el Centro de Psicología y Psiquiatría Infantil en el que se realizaron varias investigaciones a partir de numerosas consultas que realizaban familiares y directivos sobre las dificultades escolares. Este centro no tuvo presupuesto oficial y quienes trabajaron allí lo hicieron ad honorem o con salarios simbólicos. Según Hackett, funcionario de la Fundación Rockefeller en la región, este consultorio se convirtió en un lugar de capacitación y referencia para quienes se dedicaban a la psiquiatría infantil, ya que esta especialidad no contaba con la formación específica en la carrera de grado de medicina. También, fue un lugar de formación regional, puesto que concurrían estudiantes de San Pablo, Colombia y Chile. En este último país Reca dictó una serie de conferencias en 1946 con la intención de propiciar la creación de un centro similar. Del mismo modo, reconocidas médicas como Sara Raijman y Carolina Tobar García, trabajaron como voluntarias (Hackett, 1944, s/p).

Estas acciones deben enmarcarse dentro de la corriente de la higiene mental la cual hacía hincapié en la prevención y en la modificación de los factores ambientales, fundamentalmente a través de indicaciones y consejos a las familias. La intervención sobre las variables ambientales, que incidían en el desencadenamiento de la enfermedad mental, podía atenuar o retardar las manifestaciones patológicas. Para ello era necesario un diagnóstico y tratamientos precoces en las formas iniciales de la enfermedad y en los niños y adolescentes (Klappenbach, 1999; Borinsky, 2005 y Talak 2005).

Las pesquisas realizadas en el Centro de Psicología y Psiquiatría Infantil apuntaron a estudiar las conductas, y las aparentes desviaciones de la salud psíquica infantil a partir de las herramientas que otorgaba la historia clínica. Esta estrategia fue central en toda la trayectoria de Reca, pues era considerada un mecanismo para detectar anomalías y luego poder seguir el tratamiento sugerido. En 1942 realizó una meticulosa investigación y luego dictó un curso dirigido a los docentes en el Colegio Libre de Estudios Superiores con los resultados obtenidos. Esta pesquisa fue publicada en 1945 por la editorial El Ateneo bajo el título La inadaptación escolar. Problemas de conducta del niño en la escuela. Este libro contó con dos ediciones más; una en 1947 y otra en 1960 en la editorial del inmigrante español Pablo García. Esta obra también fue destacada por los funcionarios de la Fundación Rockefeller a la hora de destacar la profusa labor que realizaba Reca en Argentina. En el pedido de beca que realizó Reca en 1945 , se anexaron las referencias de este libro y una de las reseñas crítica que contó el material. ${ }^{2}$

A diferencia de su tesis doctoral, en esta experiencia, su mirada no se centró en la observación de conductas que podrían conducir a la delincuencia; sino 
que su interés estuvo puesto en reformar el sistema educativo "normal y anormal". Las maestras debían concurrir al seminario con un informe realizado a partir de su experiencia con algún alumno con problemas de conducta. El curso tuvo dos partes; una práctica, que consistió en relevar los datos familiares y la realización de exámenes médicos psicológicos y psiquiátricos. En la segunda parte, se analizó la información obtenida para detectar las causas que podrían haber alterado el desarrollo infantil y desde esta plataforma poder diseñar algún tratamiento para subsanar "la inadaptación escolar" (Reca, 1944, p.7-9). Reca proponía dividir las causas de la inadaptación escolar en biológicas, inherentes al individuo, y extrínsecas, o ambientales. Dentro de las primeras, se detenía en los casos de "anormalidad mental" vinculadas con alteraciones congénitas y heredadas. La "adaptación" de esos niños era posible solo si se modificaban las condiciones externas por medio de la creación de escuelas adecuadas, la orientación profesional tanto a los familiares como a los docentes y la psicoterapia. Dentro de las segundas, el medio ambiente, incluía el lugar geográfico, el clima, las características familiares, los sentimientos hacia el infante, la alimentación y las relaciones entabladas en la escuela. Esta variedad de estímulos influía en la estructuración de la personalidad. En este complejo arco de factores, nos detendremos en los vinculados con el rol de la escuela y el magisterio.

Reca, planteaba que era perentoria la necesidad de generalizar la organización de clases y establecimientos especiales. Si bien existían las escuelas al aire libre y las colonias de vacaciones para los "niños débiles", éstas no alcanzaban para abarcar la variedad de problemáticas existentes en la infancia. Además, postuló la necesidad que en las escuelas se diseñaran programas de estudios "dúctiles"; dado que los programas rígidos no permitían conocer "las peculiaridades y las aptitudes personales". Era crítica en torno a la concepción binaria presente en las escuelas para evaluar el intelecto (inteligencia o torpeza) o la personalidad (buena o mala). Este criterio de diferenciación dual, no tenía en cuenta, según ella, el origen de la conducta y tendía a implementar recursos educativos correctivos inadecuados que potenciaban los problemas previos. Un ejemplo que desarrolló en torno a cómo la rigidez en los sistemas educativos podía colaborar en la agudización de los trastornos fue el estudio sobre la zurdera. Según ella la imposición de escribir con la mano derecha, al ingresar a la escuela, provocaba dificultades en el aprendizaje de la escritura, la lectura e incluso a diversos trastornos motores. Lo que salía de lo común se consideraba como desviado. En oposición a esta idea, Reca planteó críticas a la habitual asociación de la zurdera con deficiencia mental y a su vinculación con una desviación. En un artículo firmado junto a Ana Matilde Montdor "La Zurdera y su relación con dificultades en el aprendizaje escolar y alteraciones en la personalidad del niño," publicado en los Archivos Argentinos de Pediatría (1951) descartaba de plano esta asociación y sugería que se restaurara la lateralidad inicialmente dominante (Reca y Montdor, 1951, p.97).

La incomprensión de maestros y directores escolares potenciaban las situaciones previas. Según ella "la equidad" en la conducta de los docentes era una herramienta importante en la educación; no obstante, reconocía que "un maestro enfermo, fatigado, angustiado, desconforme, resentido, ignorante, dogmático, no podrá tener siempre una conducta equilibrada con respecto a sus alumnos" (Reca, 1944, p.7). La escuela tenía, para Reca, un poder transformador enorme en la medida que, si bien no podía anular los efectos de "la vida pasada", podía restablecer el "perdido equilibrio" (Reca, 1944, p.9). En este sentido, "el tratamiento y la prevención de la inadaptación" debía contar con instituciones que contemplaran dichas necesidades $y$, de esta forma, limitar el impacto perjudicial que pudiera acarrear el medio social desfavorable. Por ejemplo, la calidad de la sociabilidad en los vecindarios podía tener efectos perjudiciales y actuar como estímulos de la "desviación" (Reca, 1944, p.9). Los espacios de sociabilización sin una orientación profesional no eran considerados por Reca como ámbitos formativos. Así pues, las actividades en la calle y la concurrencia al cine no debían ser fomentados, porque en esos lugares, potencialmente, se podrían desarrollar conductas consideradas inadecuadas.

Las escuelas especiales serían los espacios adecuados para los niños con deficiencias de la inteligencia o "anormalidad" de carácter que pudieran ser educables y adaptables (Reca, 1944, p.153). La "anormalidad" era definida como la incapacidad de adaptarse normalmente a la vida social: es decir, si no mediaban cuidados especiales, no se podía aprovechar la enseñanza impartida en la escuela y esto lo conduciría a presentar irregularidades de conducta o de carácter que podían llevar a la delincuencia (Reca, 1944, p.529). Abandono, anormalidad y delincuencia eran vistas por Reca como un todo. A partir de esta evaluación, el Estado debía intervenir por medio del financiamiento de escuelas especiales, dado que la "anormalidad" debía ser considerada como problema social 
(Reca, 1944, p. 153). La escuela era un espacio auxiliar a la familia y se debía reorientar para educar y atender al niño en forma adecuada. Los establecimientos deberían ser semi-internados, y contar con organización médica, educación común, física y manual (adaptada a las necesidades particulares de cada caso), y servicio social. Según Reca siempre era posible hallar tipos de tareas adecuadas y se inclinaba por trabajos manuales y faenas rurales, que los alumnos pudieran realizar con perfecta idoneidad: "Estos niños poseen aptitudes y predilecciones por determinadas actividades que son capaces de desempeñar con eficacia si tienen la instrucción necesaria" (Reca, 1944, p.221). En este sentido era partidaria de estimular espacios educativos diferenciados para potenciar el desarrollo de aptitudes adecuadas. Asimismo, reconocía que el condicionante social colaboraba en la futura inserción social: "Si la familia es pobre, falta de consejo y enseñanza sobre la naturaleza de las singularidades que observa en el niño, atribuye casi siempre su falta de progreso y escaso aprovechamiento a voluntaria inatención y pereza, y lo castiga mas o menos duramente. El niño puede presentar luego problemas de conducta mucho más graves y hasta llegar al delito, no por innatas tendencias perversas, sino porque su vida carece de elementos de satisfacción y estimulo al alcance de sus aptitudes" (Reca, 1944, p.222).

\section{b. El desempeño de Reca en la Dirección de Maternidad e Infancia}

En 1936 se sancionó la Ley № 12.341 que instauró la Dirección de Maternidad e Infancia dependiente del DNH. Su finalidad fue la de propender al perfeccionamiento de las generaciones futuras por el cultivo armónico de la personalidad del niño en todos sus aspectos, combatiendo la morbimortalidad infantil en todas sus causas y amparando a la mujer en su condición de madre o futura madre. Sus objetivos fueron la asistencia preconceptivas, del embarazo y del parto, la vigilancia del niño desde su nacimiento a través de fichas sanitarias individuales, la lactancia materna, la alimentación racional y la protección de los niños necesitados (Biernat y Ramacciotti, 2013).

En 1937 se reglamentó el decreto que determinó las divisiones en las que se organizó la Dirección y las responsabilidades de cada una de ellas: la División de Higiene Social de la Infancia, encargada de hacer investigaciones y de organizar la educación popular sanitaria; la División de Eugenesia, Maternidad y Primera Infancia ocupada de la creación, dirección técnica y vigilancia de las instituciones o servicios de atención de la madre y el niño; la División de Edad pre-escolar, escolar y adolescencia abocada a la aplicación de las medidas que se establezcan para su protección física, moral y social; la División de Infancia Abandonada, Enfermos y Anormales; la División de Inspección y Legislación ocupada del control, técnico y administrativo, de todas las instituciones, oficiales y privadas, encargadas de la asistencia y protección maternal e infantil, salvo las que dependieran de la Sociedad de Beneficencia, ya que sus instituciones habían quedado fuera del contralor de esta dependencia estatal (Biernat y Ramacciotti, 2013, p. 115 y 117).

Reca fue designada jefa de la "División Infancia Abandonada, Enfermos y Anormales", luego denominada "Infancia Abandonada, Enfermos y Anormales" y más tarde convertida en "División de Segunda Infancia". Los reiterados cambios de denominación de la repartición estuvieron ligados a las modificaciones de sus áreas de intervención y a los conflictos interinstitucionales. En línea con lo que plantea Claudia Daniel (para el caso de la médica Adela Zauchinger quien ocupara un cargo en el Departamento Nacional de Higiene) es probable que el ingreso de Reca a la función pública se haya concretado a través de uno de los mecanismos de reclutamiento del personal técnico del Estado más usual de la época: los contactos personales y, especialmente, los nexos establecidos con profesores universitarios que trabajaban en algunas de esas reparticiones públicas (Daniel, 2012, p. 92). Sus vínculos con Zwanck -jurado de su tesis, referente en el área de la Higiene Social, profesor del curso de Visitadoras de Higiene e integrante de la Comisión Municipal Honoraria para la aplicación de la ordenanza sobre "Infancia Abandonada, Anormal y Delincuente" (1934)- haya sido un acicate para su nombramiento.

Este cargo rentado, que lo ejercía en el turno tarde, luego de sus obligaciones en el Centro de Psicología y Psiquiatría Infantil, Reca impulsó tareas de investigación, tratamiento y prevención de situaciones sociales acuciantes que afectaban al binomio madre e hijo. Entre noviembre de 1937 y diciembre de 1938, promovió un relevamiento meticuloso del funcionamiento de 32 instituciones de la Capital Federal destinadas a la asistencia de "la infancia desvalida o abandonada" (Reca, 1938, p. 823). Esta indagación fue publicada en el Boletín Sanitario del Departamento Nacional de Higiene. La misma permitió conocer las características del funcionamiento de las instituciones y las condiciones sanitarias y educativas de 3.187 infantes "desvalidos" de los cuales el 53 \% eran niñas. Entre los aspectos más relevantes se destacaron los problemas sanitarios que acarreaban una falta de alimentación 
adecuada y las fallas higiénicas generales de los establecimientos: insuficiencia de sol, luz y ventilación, hacinamiento en los dormitorios, falta de cuidado y vigilancia individual, carencia de estímulos afectivos (Reca, 1938, p. 845). Además, se señaló la inexistencia de un plan metódico de educación física, aspecto considerado relevante para lograr cuerpos sanos y fuertes para el futuro, y la ausencia de un plan de observación psicológica. La propuesta de Reca, luego de este minucioso relevamiento cuantitativo y cualitativo, fue que era perentorio organizar un sistema "racional de cuidado de la salud"; la infancia "en situación de abandono" debía ser protegida dado que, según ella, el "abandono, anormalidad y delincuencia" estaban directamente relacionadas. El abandono podría ser revertido en la medida que se realizara una obra de investigación y de divulgación popular en materia de higiene social y mental.

Por tal motivo, la Dirección de Maternidad e Infancia debería abocarse al tratamiento, a la prevención y a la investigación del "problema del abandono y la anormalidad" y era el Estado que no solo debía subsidiar a dichas instituciones, de hecho, lo hacía para el $90 \%$ de ellas, sino que tenía la responsabilidad de imponer normas de funcionamiento y controlar su aplicación. Esta indagación fue pensada como un primer paso para delinear, a futuro, exámenes médicos periódicos entre grupos poblacionales más amplios $y$, de esta forma, pretendía ser un insumo, con basamento empírico, para planificar políticas sociales de mayor envergadura. ${ }^{3}$

Este metódico trabajo sobre asilos se interrumpió debido a las disputas interministeriales que se produjeron entre el Ministerio del Interior, de quien dependía, y el Ministerio de Justicia e Instrucción Pública. El Patronato Nacional de Menores interpuso un recurso administrativo, porque se consideraba que la novel agencia sanitaria avanzaba sobre atribuciones consideradas propias y sobre poblaciones de su injerencia. Esta superposición de atribuciones fue resuelta por el decreto 5.520, del 15 de junio de 1938, que estableció una nueva reglamentación. Se crearon nuevas divisiones de la Dirección y las existentes hasta el momento sufrieron modificaciones. La División de Eugenesia, Maternidad y Primera Infancia pasó a ocuparse únicamente de Eugenesia y Maternidad; se crearon las divisiones de Primera Infancia (para niños de hasta 2 años y medio de edad) y de Segunda Infancia (para los de edad preescolar); desapareció la División Edad Preescolar, Escolar y Adolescencia; las divisiones Infancia Abandonada, Enfermos y Anormales y la de
Servicio social, se concentraron en una sola: Niños Enfermos, Anormales y Necesitados; a cargo de Reca. La Dirección de Maternidad e Infancia solo podría intervenir en poblaciones menores de seis años, las problemáticas de quienes superaran esta edad serían materia de interés de las instituciones dependientes del Ministerio de Justicia e Instrucción Pública (Biernat y Ramacciotti, 2013, p. 120-121).

En la División de Segunda Infancia, nueva denominación del organismo estatal, Reca impulsó otra investigación entre 1939 y 1944. En esta oportunidad se centró en el relevamiento de jardines de infantes e instituciones similares de la Capital Federal (Briolotti, 2016, p.1086). En estos establecimientos se realizó un examen médico y psíquico a los niños y se realizaron 2.500 encuestas sociales a las familias. El objetivo, en línea con lo planeado en su anterior pesquisa, fue conocer las necesidades sociales para poder diseñar un plan de acción. Esta rigurosa pesquisa fue publicada en el Boletín Sanitario del Departamento Nacional de Higiene y en otras revistas académicas tales como Anales de la Sociedad de Puericultura de Buenos Aires y en la Revista de Pediología. En 1940, contó con un eco favorable en el Primer Congreso Nacional de Puericultura allí expresó la importancia de organizar y cuidar el desarrollo físico del niño en edad preescolar; los jardines de infantes y las escuelas maternales tenían un rol destacado para cumplir esta misión. Dicho voto tuvo su fundamento inmediato en la indagación impulsada por Reca y fue presentada en el modo de relato oficial en dicho encuentro bajo el título "Desarrollo psíquico en la primera y en la segunda infancia". Este trabajo tuvo repercusiones en la prensa periódica tal como La Prensa. Es decir que, los saberes técnicos y políticos tuvieron un espacio de difusión en los medios de comunicación y, en este sentido, ganaban notoriedad pública al mismo tiempo que "educaban" a la sociedad en la identificación de los problemas y en la búsqueda de soluciones. Es probable que con esta amplia circulación de sus ideas se intentara lograr mayor difusión de sus propuestas políticas y, de esta forma, lograr legitimidad y consenso entre la comunidad científica y la sociedad civil, con el fin de evitar recambios institucionales, recortes presupuestarios y mayor estabilidad institucional.

En línea con sus anteriores trabajos Reca fue muy crítica ante la realidad que describía. Registró grandes falencias en cuanto la formación del personal: "La preparación especial en materia de educación preescolar es excepcional y más aún lo es en asistencia social. Ninguna institución cuenta con los servicios técnicos 
de asistentes o visitadoras sociales. Las instalaciones no tienen edificios propios y no están organizados según los lineamientos que determina la arquitectura escolar" (Reca, 1940, p.14). Dentro de las deficiencias sanitarias se destacaron los problemas en la dentición asociados a las características del medio ambiente y a la alimentación, cuantitativamente y cualitativamente, defectuosa. Según Reca todas estas cuestiones se podían modificar si se impulsara una acción curativa y preventiva tanto en las instituciones como en las familias. La mencionada Ley 12.341 habilitaba a la Dirección de Maternidad e Infancia a controlar a los jardines de infantes e instituciones similares; pero el presupuesto no le asignaba partidas especiales $y$, sin ellos "las disposiciones escritas no podrán cuajar en hechos" (Reca, 1940, p. 44).

Desde este organismo estatal, Reca defendió la importancia de estudiar la valoración psíquica y el estudio de la personalidad en la infancia para poder diagnosticar tanto las deficiencias como las aptitudes desde una temprana edad. Con la elaboración de minuciosos registros, cualitativos y cuantitativos, demostró que una acción educativa organizada podría reducir las deficiencias del medio, las carencias económicas y culturales. A partir de esta observación se diferenciaba de muchos de sus colegas quienes promulgaban la existencia de una "criminalidad innata" que solo podría ser resuelta por una "reforma moral". Esta comprobación la llevó a solicitar una activa acción pública de higiene mental y estimular el desarrollo infantil por medio de la creación de instituciones de basadas en modernos lineamientos técnicos, además de subsidiar y controlar a los establecimientos privados. Con similar énfasis, reclamó la elaboración de estudios similares en el interior del país.

Con la llegada de Juan Domingo Perón a la presidencia en 1946, Reca mantuvo su cargo, continuó con su trabajo, y pudo sumar a su investigación el análisis de la situación en el Interior. En su artículo "Estudio sobre algunas condiciones de vida del niño de 2 a 6 años en la Capital y en el interior del país" publicado en la revista de la Secretaría de Salud Pública Archivos de la Secretaría de Salud Pública (1948), reconoció que, si bien las condiciones económicas habían mejorado, para muchas familias aún existían situaciones sociales caracterizadas por el hacinamiento, la promiscuidad y la escasa formación escolar. Cuestiones propensas de ser beneficiadas por medio de una activa acción estatal que promoviera la creación de viviendas populares, de centros educativos y re- creativos adecuados (Reca, 1948, p.77). A diferencia con su anterior investigación sobre los Asilos, la que realizó sobre los jardines de infantes en Capital Federal y la de las poblaciones entre 2 a 6 años en el interior del país, las personas que colaboraron en el relevamiento y sistematización de información quedaron totalmente invisibilizadas en cuanto a sus colaboraciones. Reca, para mediados del siglo XX, ya se había convertido en una figura con peso propio que le permitió, no ser removida de su cargo a pesar del recambio político, tener una posición jerárquica destacada e invisibilizar las marcas de sus colaboradores más cercanos en sus publicaciones.

Este cargo lo ejerció hasta 1948, momento en el cual obtuvo otra beca para concurrir a los EE.UU. por medio de la ayuda otorgada por la Fundación Rockefeller. Como adelantamos, el vínculo de Reca con dicha institución filantrópica fue en muy buenos términos. Además, fue una intermediadora entre muchas de sus colegas (Ej. Sara Reijman) y Hackett para acceder a becas en EE.UU. auspiciadas por la Fundación. La admiración de Hackett a la obra encarada por Reca en Buenos Aires fue destacada en varias oportunidades en la medida que la señalaba como una referente en la construcción de la psiquiatría infantil, señaló su capacidad de trabajo y su dedicación a las tareas emprendidas sin un salario de acuerdo a sus responsabilidades.

\section{REFLEXIONES FINALES}

En este artículo se reflexionó sobre la trayectoria de la médica Telma Reca, con particular énfasis a sus aportes sobre la educación "normal y anormal" y su mirada sobre cómo las intervenciones profesionales en los organismos estatales podrían modificar las condiciones sociales y, de esta forma, mitigar los efectos perniciosos de la modernización social que se expresaba en el incremento de la delincuencia juvenil y la criminalidad. Dentro de las referencias analizadas mantuvo un perfil crítico de las acciones implementadas en materia de educación y salud; no obstante, mantuvo la postura que las condiciones sociales disruptivas se podrían revertir con activas políticas estatales. Es en este sentido, que podemos ubicar a Reca como una intelectual portadora de un saber general crítico e independiente de las ideas hegemónicas de la época.

De esta forma, este trabajo permite reflexionar sobre el papel de las mujeres dentro de las profesiones sanitarias. Sabido es que el papel de los médicos recibió visibilización y abundantes referencias analíticas 
desde diferentes marcos disciplinares. Empero, el papel de Telma Reca, en la primera mitad del siglo XX, ha sido más fragmentario; a pesar de que fue una de las referentes de la higiene mental en Argentina y su tesis doctoral logró cuestionar el peso de la teoría lombrosiana. En este sentido, nos parece interesante no solo dar cuenta de sus ideas sobre el rol de la educación sino cómo sus aportes en la ciencia lograron, con su producción académica, cuestionar las ideas vigentes sobre la delincuencia y la anormalidad.

Si bien en sus obras no planteó un lugar diferente para las mujeres profesionales; impulsó su inclusión laboral dentro de las agencias estatales en su rol tradicional de asistencia y subordinación hacia el varón. Uno de los aspectos a destacar es que si bien algunas mujeres pudieron acceder al desarrollo de una vida universitaria y profesional; Reca tendió a colocarlas en los puestos que su "naturaleza femenina" las condicionaba. Es decir, a pesar de que si bien la propia práctica profesional de Reca cuestionaba el rol adjudicado tradicionalmente a las mujeres. Sus críticas no alcanzaban a discutir el lugar de la mujer como ama de casa y el varón proveedor y la definición de la identidad femenina en función de la maternidad. Así pues, en sus escritos no se encuentra una ruptura en torno a la capacidad de las mujeres para desempeñarse en el ámbito doméstico y, en el terreno profesional, para desenvolverse como, maestras, visitadoras sociales, enfermeras.

En este sentido, no nos llama la atención que la Dirección de Maternidad e Infancia haya recaído en Reca, pues dichas temáticas se las asociaba a las supuestas dotes atribuidas a la sensibilidad femenina. Lo que es interesante es cómo a partir de este estamento burocrático implementó una acción de recopilación de datos sobre instituciones asilares y educativas, sistematizó y analizó la información y produjo informes técnicos en los cuales combinó registros cuantitativos y cualitativos que fueron difundidos en publicaciones científicas de la época. Este insumo del Estado, con un marcado sesgo técnico, fue entendido como la vía para impulsar políticas públicas más eficaces y mejorar la profesionalización del personal a cargo. Si bien las injerencias del organismo que dirigió sufrieron recortes de atribuciones y cambios en la dependencia interministeriales, Reca se reubicó en su rol y arremetió con una nueva pesquisa en la que, utilizando su experiencia previa, relevó y sistematizó información sobre las condiciones de las instituciones de jardines preescolares en la ciudad de Buenos Aires y en el Interior.

Su prolífica producción y su multifacético accionar profesional da cuenta de la forma que logró adquirir las herramientas del quehacer científico: presentaciones a becas internacionales, publicaciones en revistas científicas, participaciones en congresos, invitaciones para dictar cursos y conferencias en el exterior, y publicación de libros en editoriales de amplia circulación y de artículos más cortos destinados a la divulgación científica. En sus trabajos no quebró los márgenes de los dominios que les eran socialmente asignados a las médicas, ya que los límites estuvieron apoyados en el reconocimiento de ciertos atributos y aptitudes consideradas naturales en la mujer en el imaginario de la época. Muchos de sus investigaciones se publicaron con su firma junto a la de colegas masculinos y femeninos o tuvo el respaldo de ellos para lograr becas o su inserción dentro del las agencias del Estado. En los momentos que logró tener un mayor posicionamiento dentro de su profesión mantuvo su autoría individual e invisibilizó las marcas de su equipo de trabajo.

\section{AGRADECIMIENTOS}

Agradezco a Ana Briolotti, Ricardo Pasolini y a José Cesano por facilitarme material bibliográfico sobre Telma Reca. Asimismo, los comentarios recibidos en diferentes momentos de esta investigación de Carolina Ferrante, Daniela Testa, Paula Caldo, Adriana Valobra, Flavia Fiorucci y los evaluadores anónimos de Asclepio fueron de mucha utilidad. Los Archivos de la Fundación Rockefeller nutrieron esta propuesta con el acceso al Fondo Documental de Lewis Wendell Hackett y Telma Reca. Este trabajo forma parte de los resultados del Proyecto N A00317 de la Universidad Nacional José C. Paz (UNPAZ), Resolución 200 del 31 de mayo de 2017 y del proyecto de Investigación de la Universidad Nacional de Quilmes "El proceso de profesionalización del cuidado sanitario. La enfermería universitaria en Argentina (1940-1970)".

\section{NOTAS}

1. Archivos de la Fundación Rockefeller (1942-1943, 19451946, 1948), University of Buenos Aires - Reca de Acosta, Telma - (Child Psychiatry), Box 3, Folder 34.
2. Véase Archivos de la Fundación Rockefeller (1945-1946), University of Buenos Aires - Reca de Acosta, Telma - (Child Psychiatry), -Box 3 Folder 34. 
3. Los resultados de esta investigación, si bien fueron firmados de manera individual por Reca, fueron parte de un trabajo colaborativo. Seis médicos realizaron las visitas de inspección y los exámenes de los niños internados en los asilos (Guillermo Bayley Bustamante; Felipe de Elizalde; Tiburcio

\section{BIBLIOGRAFÍA}

Aguilar, Lucía (2014), El hogar como problema y como solución. Una mirada genealógica de la domesticidad a través de las políticas sociales. Argentina 1890-1940, Buenos Aires, Centro Cultural de la Cooperación.

Ariès, Philippe (1960), El niño y la vida familiar en el Antiguo Régimen, Madrid, Taurus.

Biernat, Carolina y Ramacciotti, Karina (2013), Crecer y multiplicarse. La política sanitaria materno-infantil argentina 19001960, Buenos Aires, Biblos.

Borinsky, Marcela (2005), "De la delincuencia infantil a los problemas de Conducta en el niño", XII Jornadas de Investigación y Primer Encuentro de Investigadores en Psicología del Mercosur, Facultad de Psicología - Universidad de Buenos Aires, Buenos Aires, pp.191-193.

Briolotti, Ana (2016), "La evaluación del desarrollo psicológico en los dispensarios de lactantes de Buenos Aires: medicina y psicología en la Argentina, 1935-1942", História, Ciências, Saúde-Manguinhos, 23 (4), pp.1077-1093.

Cammarota, Adrián (2016), "Saberes médicos y medicalización del sistema escolar en Argentina (1924-1940)", Revista Pilquen, 19 (3), pp. 33-51. Universidad Nacional del Comahue.

Carli, Sandra (1992), "El campo de la niñez. Entre el discurso de la minoridad y el discurso de la educación nueva". En Puigrós, Adriana (ed.), Escuela, democracia y orden (19161943), Buenos Aires, Galerna.

Cesano, José (2015), "La medicalización de la delincuencia infantil: una aproximación a la obra de Telma Reca". En Reca, Telma, Delincuencia infantil en los Estados Unidos y en la Argentina, Córdoba, Buena Vista.

Dagfal, Alejandro (2009), Entre París y Buenos Aires: Ia invención del psicólogo, 1942-1966, Buenos Aires, Paidós.

Daniel, Claudia (2012), "Contar para curar: estadísticas y comunidad médica en Argentina, 1880-1940", História, Ciências, Saúde - Manguinhos, 19 (1), pp. 89-114.

Donzelot, Jacques (1998), La Policía de las Familias, 2a ed., Valencia, España, Pre Textos.

Fendrik, Silvia (2006), Psicoanalistas de niños. La verdadera historia. 3. Arminda Aberastury y Telma Reca, Buenos Aires, Letra Viva.
Padilla, Teodoro Ruiz Moreno, Generoso Schiavone y Stornelli). También colaboraron siete asistentes sociales. Ellas levantaron la encuesta del censo, e investigaron los antecedentes sociales de los niños internados (Bladó; Escardó; Elena Gualtruzi; Iriart; Itzcovitz; Dora Lacroix y Zapata).

Guy, Donna (2008), Women Create the Welfare State: Performing Charity, Creating Rights in Argentina 1880-1955, Durham, Duke University Press.

Hackett, Lewis (1944), Diario, 21 de Septiembre de 1944 y 23 de octubre $1944, \mathrm{~s} / \mathrm{p}$.

Klappenbach, Hugo (1989), "Higiene mental en las primeras décadas del siglo. Principales lineamientos y fundamentos ideológicos", Primer Anuario de Investigaciones, Buenos Aires, Universidad de Buenos Aires, pp. 189-195.

Klappenbach, Hugo (1999), "El movimiento de la higiene mental y los orígenes de La Liga Argentina de Higiene mental", Temas de Historia de la Psiquiatría Argentina, (10), pp. 3-17.

Lardies González, Julio (1974), “Médicas argentinas con aportes sociológicos", Archivos de Historia de la Medicina Argentina, (9), pp. 26-29.

Lionetti, Lucía (2011), "Discursos, representaciones y prácticas educativas sobre el cuerpo de los escolares. Argentina en las primeras décadas del siglo XX", Cuadernos de Historia, 34, pp. 31-52, Departamento de Ciencias Históricas, Universidad de Chile.

Lorenzo, María Fernanda (2016), Que sepa coser, que sepa bordar, que sepa abrir la puerta para ir a la universidad. Las académicas de la universidad de Buenos Aires en la primera mitad del siglo xx, Buenos Aires, Eudeba.

Norando, Verónica (2016), Relaciones de género y militancia. El comunismo dentro de la Unión Obrera Textil y las trabajadoras. 1936-1946, Tesis de Doctorado, Buenos Aires: Universidad de Buenos Aires.

Pasolini, Ricardo (2013), Los marxistas liberales. Antifascismo y cultura comunista en la Argentina del siglo XX, Buenos Aires, Sudamericana.

Plotkin, Mariano (2003), Freud en las pampas, Orígenes y desarrollo de una cultura psicoanalítica en la Argentina (19101983), Buenos Aires, Sudamericana.

Queirolo, Graciela (2013), "Género y sexualidad en tiempos de males venéreos (Buenos Aires, 1920-1940)", Revista Nomadías, (17), pp. 67-87.

Ramacciotti, Karina y Valobra, Adriana (2011), "Modernas esculapios: acción política e inserción profesional, 1900-1950". 
En Scarzanella, Eugenia y Lizette, Jacinto (eds.), Género y ciencia: hombres, mujeres e investigación científica en América Latina, Siglos XVIII-XX, Colección Estudios AHILA de Historia Latinoamericana, 8, Madrid/Frankfurt, Asociación de Historiadores Latinoamericanistas Europeos, pp. 15-33.

Ramacciotti, Karina y Testa, Daniela (2014), “La niñez anormal. Discurso médico sobre la infancia, 1900-1950", Inclusiones, no especial, pp. 226-248, [en línea], disponible en: http://hdl. handle.net/11181/4500 [consultado el 15/3/2017].

Reca, Telma (1932a), De la vida norteamericana, Buenos Aires, M. Gleizer Editor.

Reca, Telma (1932b), Delincuencia infantil en los Estados Unidos y en la Argentina, Tesis de Doctorado, Universidad de Buenos Aires, Talleres Gráficos de la Penitenciaría Nacional.

Reca, Telma (1937). "Nuevas perspectivas de la terapéutica educacional en psicopatalogía infantil", Revista de Pediología, 1 (8), pp. 20-25.

Reca, Telma (1938), "Problemas médicos sociales de la asistencia de niños necesitados en los Asilos de Buenos Aires", Boletín Sanitario del Departamento Nacional de Higiene, Año II, pp. 823-849.

Reca, Telma (1940), Educación y asistencia de la edad preescolar en la ciudad de Buenos Aires, pp. 3-44.

Reca, Telma (1944), La inadaptación escolar. Problemas de conducta del niño en la escuela, Buenos Aires, Editorial El Ateneo.

Reca, Telma (1948), "Estudio sobre algunas condiciones de vida del niño de 2 a 6 años en la Capital y en el Interior del país", Archivos de la Secretaría de Salud Pública, III (14), pp. 64-77.

Reca, Telma y Montdor, Ana Matilde (1951), "La zurdera y su relación con las dificultades en el aprendizaje escolar y al- teraciones en la personalidad del niño", Archivos Argentinos de Pediatría, XXXVI, pp. 96-108.

Reca, Telma y Zwanck, Alberto (1934), “Comedores Escolares. Resultados de una encuesta", La Semana Médica, (6).

Sánchez, Norma (2007), La Higiene y los higienistas en la Argentina (1880-1943), Buenos Aires, Sociedad Científica Argentina.

Stagnaro, Juan Carlos (2011), “Carolina Tobar García. Psiquiatría infantil, pedagogía, criminología y prevención”. En Sánchez Norma (comp.), Historia de la niñez en la Argentina. Una mirada médica y sociocultural (1880-1930), Buenos Aires, Dunken.

Stagno, Leandro (2011), "Los tribunales de menores en la Argentina. Antecedentes internacionales e iniciativas nacionales". En Cosse, Isabella; Llobet, Valeria; Villalta, Carla; Zapiola, María Carolina (eds.), Infancias: Políticas y saberes en Argentina y Brasil, Buenos Aires, Editorial Teseo, pp. 335-364.

Talak, Ana (2005), “Eugenesia, e Higiene mental: Usos de la psicología en Argentina (1900-1940)". En Miranda, Marisa y Vallejo, Gustavo (eds.), Darwinismo social y eugenesia en el mundo latino, Buenos Aires, Siglo XXI Editora Iberoamericana, pp. 563-600.

Valobra, Adriana (2012), "Recorridos, tensiones y desplazamientos en el ideario de Alicia Moreau", Revista Nomadías, (15), pp. 139-169.

Valobra, Adriana (2015), "Formación de cuadros y frentes populares: las mujeres en el Partido Comunista de Argentina, 1935-1951", Revista Izquierdas, (23), pp. 127-156.

Zapiola, María Carolina (2010), "La Ley de Patronato de Menores de 1919: ¿una bisagra histórica?”. En Lionetti, Lucía y Míguez, Daniel (comps.), Las infancias en la historia argentina. Intersecciones entre prácticas, discursos e instituciones (1890-1960), Buenos Aires, Prohistoria, pp. 117-132. 